Research Article

\title{
General Traveling Wave Solutions of Nonlinear Conformable Fractional Sharma-Tasso-Olever Equations and Discussing the Effects of the Fractional Derivatives
}

\author{
Kai Fan $\mathbb{D}^{1,2,3,4}$ Rui Wang $\mathbb{D}^{2,5}$ and Cunlong Zhou $\mathbb{D}^{2,3}$ \\ ${ }^{1}$ Engineering Research Center of Heavy Machinery Ministry of Education, Taiyuan University of Science and Technology, \\ Taiyuan 030024, China \\ ${ }^{2}$ School of Mechanical Engineering, Taiyuan University of Science and Technology, Taiyuan 030024, China \\ ${ }^{3}$ Shanxi Provincial Key Laboratory of Metallurgical Device Design Theory and Technology, Taiyuan University of Science \\ and Technology, Taiyuan 030024, China \\ ${ }^{4}$ School of Applied Science, Taiyuan University of Science and Technology, Taiyuan 030024, China \\ ${ }^{5}$ School of Mechanical and Equipment Engineering, Hebei University of Engineering, Handan 056107, China
}

Correspondence should be addressed to Kai Fan; 2014279@tyust.edu.cn, Rui Wang; wr1396595@163.com, and Cunlong Zhou; zcunlong@tyust.edu.cn

Received 16 March 2021; Revised 25 April 2021; Accepted 11 May 2021; Published 25 May 2021

Academic Editor: Kamyar Hosseini

Copyright (c) 2021 Kai Fan et al. This is an open access article distributed under the Creative Commons Attribution License, which permits unrestricted use, distribution, and reproduction in any medium, provided the original work is properly cited.

The exact traveling wave solution of the fractional Sharma-Tasso-Olever equation can be obtained by using the function expansion method, but the general traveling wave solution cannot be obtained. After transforming it into the Sharma-Tasso-Olever equation of the integer order by the fractional complex transformation, the general solution of its traveling wave is obtained by a specific function transformation. Through parameter setting, the solution of the kinked solitary wave is found from the general solution of the traveling wave, and it is found that when the two fractional derivatives become smaller synchronically, the waveform becomes more smooth, but the position is basically unchanged. The reason for this phenomenon is that the kink solitary wave reaches equilibrium in the counterclockwise and clockwise rotation, and the stretching phenomenon is accompanied in the process of reaching equilibrium. This is a further development of our previous work, and this kind of detailed causative analysis is rare in previous papers.

\section{Introduction}

Because of many phenomena, integer-order differential equations cannot be well described, which makes fractional nonlinear differential equations have research significance. As an effective mathematical modeling tool, it is widely used in the mathematical modeling of nonlinear phenomena in biology, physics, signal processing, control theory, system recognition, and other scientific fields. The widely studied fractional Sharma-Tasso-Olever (STO) equation in space and time [1-4]

$$
\begin{gathered}
\left.\frac{\partial^{\alpha} u}{\partial t^{\alpha}}+3 \rho u^{2} \frac{\partial^{\beta} u}{\partial x^{\beta}}+3 \rho\left(\left[\frac{\partial^{\beta} u}{\partial x^{\beta}}\right]^{2}+u \frac{\partial^{2 \beta} u}{\partial x^{2 \beta}}\right)\right) \\
+\rho \frac{\partial^{3 \beta} u}{\partial x^{3 \beta}}=0,0<\alpha \leq 1,0<\beta \leq 1 .
\end{gathered}
$$

In equation (1), if $\alpha=\beta$, we get [5]

$$
\left.\frac{\partial^{\alpha} u}{\partial t^{\alpha}}+3 \rho u^{2} \frac{\partial^{\alpha} u}{\partial x^{\alpha}}+3 \rho\left(\left[\frac{\partial^{\alpha} u}{\partial x^{\alpha}}\right]^{2}+u \frac{\partial^{2 \alpha} u}{\partial x^{2 \alpha}}\right)\right)+\rho \frac{\partial^{3 \alpha} u}{\partial x^{3 \alpha}}=0
$$

In equation (1), if $\beta=1$, we get the time-fractional STO equation $[6,7]$

$$
D_{t}^{\alpha} u+3 \rho u^{2} u_{x}+3 \rho u_{x}^{2}+3 \rho u u_{x x}+\rho u_{x x x}=0,0<\alpha \leq 1
$$

In equations (1)-(3), $u=u(x, t)$ is the function to be solved, $\rho$ is an arbitrary real parameter, and $\alpha, \beta$ are conformable fractional derivatives. 
Recently, we have looked at some papers that obtain the exact solutions of the traveling wave of fractional-order equations and have illustrated some solitary wave solutions contained in the exact traveling wave solutions. These articles used different methods to obtain a large number of accurate traveling wave solutions in various forms. In order to better understand our work, we present the definitions and properties of the conformable fractional derivative as follows.

Given a function $f:[0, \infty] \rightarrow R$. Then, the conformable fractional derivative of $f$ of order $0<\alpha<1$ is defined as [8]

$$
T_{\alpha}(f)(t)=\lim _{\varepsilon \rightarrow 0} \frac{f\left(t+\varepsilon t^{1-\alpha}\right)-f(t)}{\varepsilon} .
$$

The conformable derivative is a fractal derivative [9]. According to the fractal derivative theory, the influence of environmental abnormal fluctuation on physical behavior is equivalent to the influence of fractal space-time transformation $[10,11]$. Several properties of conformable fractional derivative definition are as follows:

$$
\begin{aligned}
& T_{\alpha}(a f+b g)=a T_{\alpha}(f)+b T_{\alpha}(g) \text {, for all } a, b \in R, \\
& T_{\alpha}(C)=0 \text {, } \\
& T_{\alpha}\left(t^{b}\right)=b t^{b-\alpha}, \text { for all } b \in R, \\
& T_{\alpha}(f g)=g T_{\alpha}(f)+\mathrm{fT}_{\alpha}(g), \\
& \text { if } f \text { is differentiable, } T_{\alpha}(f)(t)=t^{1-a} \frac{d f}{d t}(t) \text {. }
\end{aligned}
$$

These properties have been proved in literature [8], and there is no need to repeat them here. The physical interpretation of the conformable fractional derivative can be found in the literature [12].

According to Theorem 2.11 (chain Rule) in literature [13], we show the following properties of consistent fractional derivatives.

$$
T_{\alpha}^{t} f(g(t))=f_{g}^{\prime}(g(t)) g^{\prime}(t) t^{1-\alpha}
$$

For more detailed knowledge, refer to literature [13]. Formula (6) is briefly proved as follows.

Proof. Set $u=t+\varepsilon t^{1-\alpha}$ in the definition, and you get

$$
\begin{aligned}
T_{\alpha}^{t} f(g(t)) & =\lim _{u \rightarrow t} \frac{f(g(u))-f(g(t))}{u-t} t^{1-\alpha} \\
& =\lim _{u \rightarrow t} \frac{f(g(u))-f(g(t))}{g(u)-g(t)} \cdot \lim _{u \rightarrow t} \frac{g(u)-g(t)}{u-t} t^{1-\alpha} \\
& =\lim _{g(u) \rightarrow g(t)} \frac{f(g(u))-f(g(t))}{g(u)-g(t)} \cdot \lim _{u \rightarrow t} \frac{g(u)-g(t)}{u-t} t^{1-\alpha} \\
& =f_{g}^{\prime}(g(t)) g^{\prime}(t) t^{1-\alpha} .
\end{aligned}
$$

Now, let us look at some of the work with the STO equation of fractional order to find the exact traveling wave solution.
In literature [1], the author used fractional complex transformation for equation (1) and then used the expfunction method to obtain its precise traveling wave solution.

In literature [2], the author used fractional complex transformation on equation (1) and then used $\left(G^{\prime} / G^{2}\right)$ -expansion method to obtain the precise traveling wave solution of equation (1).

In literature [3], the author used fractional complex transformation on equation (1) and then used the novel $\left(G^{\prime} / G\right)$-expansion method to obtain the exact traveling wave solution of equation (1); the generalized Kudryashov method was also used to obtain the precise traveling wave solution of equation (1).

In literature [4], the author used the improved $\left(G^{\prime} / G\right)$ -expansion method to get the exact traveling wave solution of equation (1) after using the complex fractional transformation.

In literature [5], the author used fractional complex transformation for equation (2), and then extended tanhcoth method was used to obtain the precise traveling wave solution of equation (2).

In literature [6], the author used fractional complex transformation for equation (3) and then used the Riccadi function expansion method to obtain the exact traveling wave solution of fractional-order equation (3).

In literature [7], the author used fractional complex transformation in equation (3) and then used a new generalized $\left(G^{\prime} / G\right)$-expansion method to obtain the precise traveling wave solution of the fractal equation (3).

Here, we have just listed some of the articles on the accurate line-wave solution to the STO equation of fractional order. It can be seen from literature [1-7] that the first step of these authors was to reduce the STO equation of the fractional order to a nonlinear ordinary differential equation by using fractional complex transformation and then to solve the reduced equation by various methods to obtain the solution of the original fractional-order equation. In addition to the methods provided in literature [1-7], there are other techniques that can be used to obtain wave solutions of different structures [14-19]. It can be seen from equations (1)-(3) that the most general equation is equation (1), so we only discuss the accurate general traveling wave solution of equation (1).

For equation (1), $\alpha, \beta$ are conformable fractional derivatives and the fractional complex transformation used is [20]

$$
u(x, t)=U(\xi), \xi=\frac{c t^{\alpha}}{\alpha}+\frac{k x^{\beta}}{\beta},
$$

where $c, k$ are parameters to be determined. Through equation (8), equation (1) can be transformed into

$$
c U^{\prime}+3 k^{2} \rho\left(U^{\prime}\right)^{2}+3 k^{2} \rho U U^{\prime \prime}+3 k \rho U^{2} U^{\prime}+k^{3} \rho U^{\prime \prime \prime}=0 .
$$

In literature [1-7], some articles integrate equation (13) once and then assume that the integral constant is zero, 
which will lead to the exact solution obtained being less general than its original one [21, 22]. Equation (9) is a thirdorder differential equation, and in general, its general solution should contain three arbitrary constants. From this perspective, the exact line-wave solutions obtained in the article [1-7] we mentioned are not general solutions, but only partial solutions. In this paper, we consider the integrability of equation (9) and combine with the fractional complex transformation to obtain the general traveling wave solution of the fractional STO equation, which is different from the exact solution obtained by using the function expansion method in known literature. As we all know, from the exact solutions of traveling waves, various forms of solutions can be obtained by choosing appropriate parameters, such as soliton solutions and periodic wave solutions. This conclusion can be obtained by bifurcation analysis of the first integral equation of equation (9) [23]. If the reader wants to know how the exact linear wave solutions of equation (9) are bifurcated and how the exact traveling wave solutions correspond to different forms of solutions, please refer to [23]. We selected from the general solution of traveling wave a kink solitary wave to fractional-order parameter change on the influence of the waveform, found that when two fractional-order parameters decrease at the same time, the kink soliton will become more smooth, but the location remains the same basic phenomenon, through the analysis found that the cause of this phenomenon is a kink soliton in the clockwise and counterclockwise to balance. Papers with such detailed analysis are rare.

\section{The General Solution of the Fractional STO Equation in Space and Time in the Traveling Wave}

Substituting equation (8) into equation (1) for transformation, we get

$$
c U^{\prime}+3 k^{2} \rho\left(U^{\prime}\right)^{2}+3 k^{2} \rho U U^{\prime \prime}+3 k \rho U^{2} U^{\prime}+k^{3} \rho U^{\prime \prime \prime}=0 .
$$

Integrate equation (10) once, and we get

$$
c U+3 k^{2} \rho U U^{\prime}+k \rho U^{3}+k^{3} \rho U^{\prime \prime}+C_{0}=0,
$$

where " $U^{\prime \prime}=d U / d \xi, C_{0}$ is the integral constant.

Considering the specific function transformation,

$$
U=\frac{k F^{\prime}}{F},
$$

where " $F^{\prime \prime}=d F / d \xi, F=F(\xi)$, we can easily get the following equation.

$$
\begin{aligned}
& U^{\prime}=k \frac{F^{\prime \prime} F-\left(F^{\prime}\right)^{2}}{F^{2}}, \\
& U U^{\prime}=k^{2} \frac{F^{\prime \prime} F^{\prime} F-\left(F^{\prime}\right)^{3}}{F^{3}}, \\
& U^{\prime \prime}=k \frac{\left(F^{\prime \prime \prime} F+F^{\prime \prime} F^{\prime}-2 F^{\prime} F^{\prime \prime}\right) F-\left(F^{\prime \prime} F-\left(F^{\prime}\right)^{2}\right) 2 F^{\prime}}{F^{3}} .
\end{aligned}
$$

Substituting equations (13) and (14) into equation (11), we get

$$
\begin{aligned}
c k \frac{F^{\prime}}{F} & +3 k^{4} \rho \frac{F^{\prime \prime} F^{\prime} F-\left(F^{\prime}\right)^{3}}{F^{3}}+\rho k^{4} \frac{\left(F^{\prime}\right)^{3}}{F^{3}} \\
& +\rho k^{4} \frac{\left(F^{\prime \prime \prime} F+F^{\prime \prime} F^{\prime}-2 F^{\prime} F^{\prime \prime}\right) F-\left(F^{\prime \prime} F-\left(F^{\prime}\right)^{2}\right) 2 F^{\prime}}{F^{3}} \\
& +C_{0}=0 .
\end{aligned}
$$

Arrange equation (15) to get

$$
k \frac{F^{\prime}}{F}+\rho k^{4} \frac{F^{\prime \prime \prime}}{F}+C_{0}=0 \Rightarrow F^{\prime \prime \prime}+\frac{c}{\rho k^{3}} F^{\prime}+\frac{C_{0}}{\rho k^{4}} F=0
$$

Equation (16) is a familiar three-differential equation, and its general solution can be easily obtained, denoted as $p=c l \rho k^{3} \neq 0, \quad q=C_{0} / \rho k^{4}$; then, equation (16) has a characteristic equation

$$
r^{3}+p r+q=0
$$

The three roots of equation (17) can be obtained by Cardin formula in the following order:

$$
r_{1}=\sqrt[3]{-\frac{q}{2}+\sqrt{\left(\frac{q}{2}\right)^{2}+\left(\frac{p}{3}\right)^{3}}}+\sqrt[3]{-\frac{q}{2}-\sqrt{\left(\frac{q}{2}\right)^{2}+\left(\frac{p}{3}\right)^{3}}},
$$

$$
r_{2}=\omega \sqrt[3]{-\frac{q}{2}+\sqrt{\left(\frac{q}{2}\right)^{2}+\left(\frac{p}{3}\right)^{3}}}+\omega^{2} \sqrt[3]{-\frac{q}{2}-\sqrt{\left(\frac{q}{2}\right)^{2}+\left(\frac{p}{3}\right)^{3}}}
$$

$$
r_{3}=\omega^{2} \sqrt[3]{-\frac{q}{2}+\sqrt{\left(\frac{q}{2}\right)^{2}+\left(\frac{p}{3}\right)^{3}}}+\omega \sqrt[3]{-\frac{q}{2}-\sqrt{\left(\frac{q}{2}\right)^{2}+\left(\frac{p}{3}\right)^{3}}}
$$

where $\omega=(-1+\sqrt{3} i) / 2$. 
(1) When discriminant $\Delta=(q / 2)^{2}+(p / 3)^{3}=0$, that is, $\left(C_{0}\right)^{2}=4 c^{3} / 27 \rho k$, the three roots of equation (17) are reduced to

$$
r_{1}=2 \sqrt[3]{-\frac{q}{2}}, r_{2}=r_{3}=\sqrt[3]{\frac{q}{2}}
$$

equation (16) has the following general solution:

$$
F(\xi)=C_{1} e^{2 \sqrt[3]{-(q / 2)} \xi}+\left(C_{2}+C_{3} \xi\right) e^{\sqrt[3]{(q / 2)} \xi}
$$

where $C_{1}, C_{2}$, and $C_{3}$ are arbitrary constants

Without loss of generality, we can assume $C_{1}$ is not equal to zero. By substituting equation (22) into equation (12) and combining with complex transformation equation (8), the general traveling wave solution of the original equation (1) is expressed as

$$
\begin{aligned}
U & =k \frac{C_{1} 2 \sqrt[3]{-(q / 2)} e^{2 \sqrt[3]{-(q / 2)} \xi}+\left(C_{2} \sqrt[3]{q / 2}+C_{3} \sqrt[3]{(q / 2)} \xi+C_{3}\right) e^{\sqrt[3]{(q / 2) \xi}}}{C_{1} e^{2 \sqrt[3]{-(q / 2)} \xi}+\left(C_{2}+C_{3} \xi\right) e^{\sqrt[3]{(q / 2)} \xi}} \\
& =k \frac{2 \sqrt[3]{-(q / 2)} e^{2 \sqrt[3]{-(q / 2)} \xi}+\left(C_{2} / C_{1} \sqrt[3]{q / 2}+C_{3} / C_{1} \sqrt[3]{(q / 2)} \xi+\left(C_{3} / C_{1}\right)\right) e^{\sqrt[3]{(q / 2) \xi}}}{e^{2 \sqrt[3]{-(q / 2) \xi}}+\left(\left(C_{2} / C_{1}\right)+\left(C_{3} / C_{1}\right) \xi\right) e \sqrt[3]{(q / 2) \xi}} .
\end{aligned}
$$

In equation (23), there are only two arbitrary constants, which is caused by the fixed arbitrary constant $C_{0}$. Without loss of generality, we could write $C_{4}=C_{2} / C_{1}, C_{5}=C_{3} / C_{1}$.

(2) When discriminant $\Delta=(q / 2)^{2}+(p / 3)^{3} \neq 0$, equation (19) has the following general solution:

$$
F(\xi)=C_{1} e^{r_{1} \xi}+C_{2} e^{r_{2} \xi}+C_{3} e^{r_{3} \xi}
$$

where $C_{1}, C_{2}$, and $C_{3}$ are arbitrary constants

Without loss of generality, we can assume $C_{1}$ is not equal to zero. By substituting equation (24) into equation (12) and combining with complex transformation equation (8), the general traveling wave solution of the original equation (1) is expressed as

$$
\begin{aligned}
U & =k \frac{C_{1} r_{1} e^{r_{1} \xi}+C_{2} r_{2} e^{r_{2} \xi}+C_{3} r_{3} e^{r_{3} \xi}}{C_{1} e^{r_{1} \xi}+C_{2} e^{r_{2} \xi}+C_{3} e^{r_{3} \xi}} \\
& =k \frac{r_{1} e^{r_{1} \xi}+\left(C_{2} / C_{1}\right) r_{2} e^{r_{2} \xi}+\left(C_{e} / C_{1}\right) r_{3} e^{r_{3} \xi}}{e^{r_{1} \xi}+\left(C_{2} / C_{1}\right) e^{r_{2} \xi}+\left(C_{3} / C_{1}\right) e^{r_{3} \xi}} .
\end{aligned}
$$

Without loss of generality, we could write $C_{4}=C_{2} / C_{1}$, $C_{5}=C_{3} / C_{1}$, and we have an arbitrary constant $C_{0}$ hidden in the parameter $q$.

\section{The Discussion and Explanation}

We observed some articles about the STO equation of fractional order and found that they more or less ignored the properties of the equation itself in the solution process, and

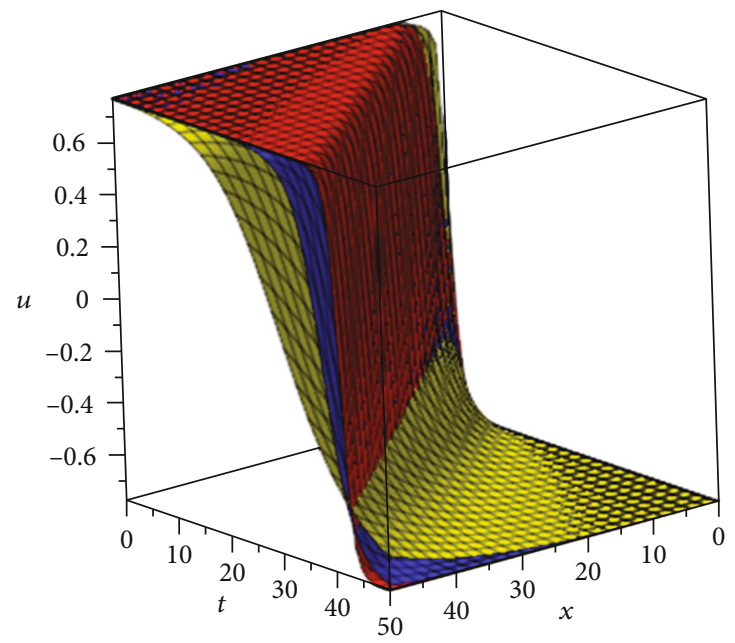

Solution (27) red: $\alpha=\beta=1$, blue: $\alpha=\beta=0.7$, yellow: $\alpha=\beta=0.4$

FIgURe 1: 3D plot of solution (27) for various values of $\alpha=\beta$, and $k=1, \rho=2, c=-1.2, C_{4}=C_{5}=1$.

the exact solution of the traveling wave obtained by various function expansion methods was not the general solution. Because in general, it makes sense that the precise general solution to equation (10) should contain three arbitrary constants. In the following part, we will find the kinked solitary wave solution from the general solution obtained in this paper and analyze the influence of the synchronous change of two fractional derivatives on the kinked solitary wave solution waveform and the reasons for this phenomenon.

Let $\xi$ in equation (25) be the expression in equation (8), namely,

$$
\xi=\frac{c t^{\alpha}}{\alpha}+\frac{k x^{\beta}}{\beta} .
$$

We take the integral constant $C_{0}$ in equation (11) as zero; then, $r_{1}, r_{2}$, and $r_{3}$ in equation (25) are valued as $r_{1}=0, r_{2}=\sqrt{-p}=\sqrt{-c / \rho k^{3}}, r_{3}=-\sqrt{-p}=-\sqrt{-c / \rho k^{3}} ;$ then, equation (25) is rewritten as

$$
\begin{aligned}
U & =k \frac{r_{1} e^{r_{1} \xi}+\left(C_{2} / C_{1}\right) r_{2} e^{r_{2} \xi}+\left(C_{3} / C_{1}\right) r_{3} e^{r_{3} \xi}}{e^{r_{1} \xi}+\left(C_{2} / C_{1}\right) e^{r_{2} \xi}+\left(C_{3} / C_{1}\right) e^{r_{3} \xi}} \\
& =k \frac{C_{4} r_{2} e^{r_{2} \xi}+C_{5} r_{3} e^{r_{3} \xi}}{1+C_{4} e^{r_{2} \xi}+C_{5} e^{r_{3} \xi}} \\
& =k \frac{C_{4} \sqrt{-c / \rho k^{3}} e^{\sqrt{-c l \rho k^{3}}\left(\left(c t^{\alpha} / \alpha\right)+\left(k x^{\beta} / \beta\right)\right)}-C_{5} \sqrt{-c / \rho k^{3}} e^{-\sqrt{-c l \rho k^{3}}\left(\left(c t^{\alpha} / \alpha\right)+\left(k x^{\beta} / \beta\right)\right)}}{1+C_{4} e^{\sqrt{-c \rho k^{3}}\left(\left(c t^{\alpha} / \alpha\right)+\left(k x^{\beta} / \beta\right)\right)}+C_{5} e^{-\sqrt{-c \rho k^{3}}\left(\left(c t^{\alpha} / \alpha\right)+\left(k x^{\beta} / \beta\right)\right)}} .
\end{aligned}
$$

In equation (27), take $k=1, \rho=2, c=-1.2, C_{4}=C_{5}=1$; then, the diagram of solution (27) of equation (1) changing with $\alpha=\beta$ is shown in Figure 1. This is the kink solitary wave solution, which is a particular solution of the general solution (25) of equation (1). When $\alpha=\beta=1$, the fractional-order STO equation degenerates into an equation of integer order. At this point, the diagram of 


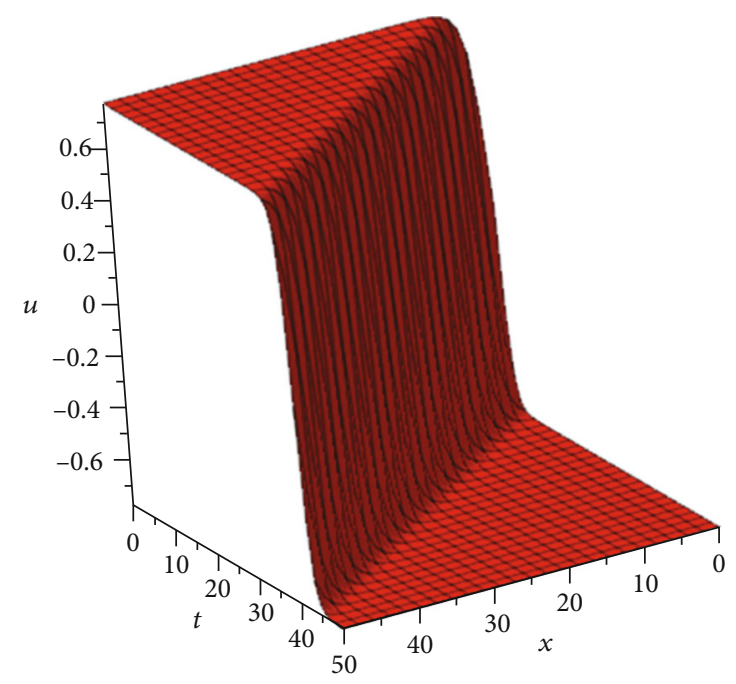

Figure 2: 3D plot of solution (27) for values of $\alpha=\beta=1$, and $k=1, \rho=2, c=-1.2, C_{4}=C_{5}=1$.

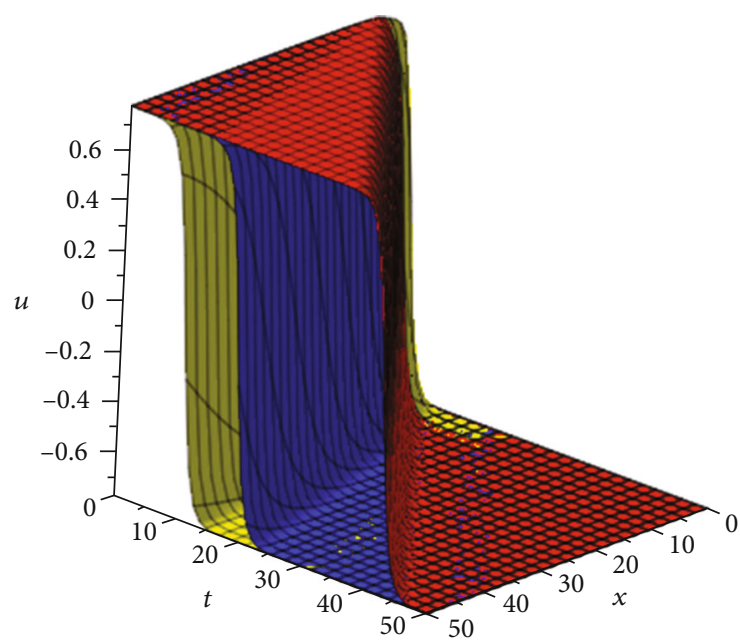

$\alpha=1$; solution (27) red: $\beta=1$, blue: $\beta=0.7$, yellow: $\beta=0.4$

FIgURE 3: 3D plot of solution (27) for various values of $\beta$, and $k=1, \rho=2, c=-1.2, C_{4}=C_{5}=1$.

equation (27) is taken from Figure 1, as shown in Figure 2. After setting values for other parameters, Figure 1 explains the perspective view of the solution (27), when the values of $\alpha=\beta$ are $1,0.7$, and 0.4 in turn.

As can be seen from Figure 1, as the fractional derivative $\alpha$ and $\beta$ become smaller synchronically, the twist of the kink solitary wave becomes smoothed, but the position of the twist of the kink solitary wave basically remains at the straight line $x=t$ on the $t-x$ plane. In order to further explain this phenomenon and promote our previous work [24], this paper conducts a more detailed study of the influence of fractional derivative on the shape of kink solitary wave in two steps. First, the value of the time fractional derivative $\alpha$ is fixed, and only the change of the spatial fractional derivative $\beta$ is observed. The result is shown in Figure 3. It can be seen from Figure 3 that the spatial fractional derivative $\beta$ values $1,0.7$, and 0.4 in turn, and the kinked solitary wave rotates at the

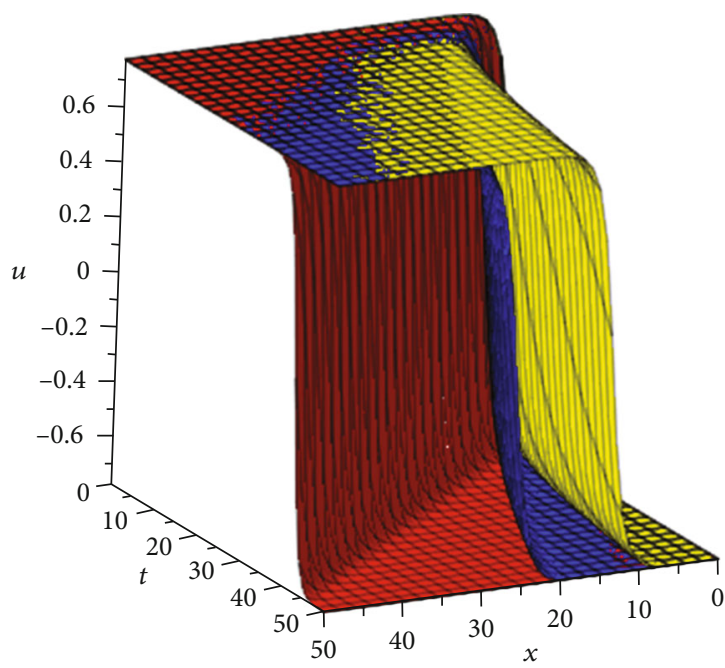

$\beta=1$; solution (27) red: $\alpha=1$, blue: $\alpha=0.7$, yellow: $\alpha=0.4$

FIgURE 4: 3D plot of solution (27) for various values of $\alpha$, and $k=1, \rho=2, c=-1.2, C_{4}=C_{5}=1$.

kinked position in the $t-x$ plane, and the direction of rotation is close to the line $t=0$ inside the $t-x$ plane. Secondly, the value of the spatial fractional derivative $\beta$ is fixed, and only the change of the time fractional derivative $\alpha$ is observed. The result is shown in Figure 4. It can be seen from Figure 4 that the time fractional derivative $\alpha$ is $1,0.7$, and 0.4 in turn, and the kinking position of the kink solitary wave in the $t-x$ plane also rotates, with the direction approaching the line $x=0$ in the $x$ - $t$ plane.

By comparing Figures 3 and 4, it is found that the spatial fractional derivative $\beta$ becomes smaller, making the kink of the kink isolated wave as shown in the figure rotate clockwise in the $t-x$ plane, while the time fractional derivative $\alpha$ becomes smaller, making the kink of the kink isolated wave as shown in the figure rotate counterclockwise in the $t-x$ plane. When the fractional derivatives change synchronically, the kinks of the isolated wave in Figure 1 reach a balance in the clockwise rotation and counterclockwise rotation, and the position of the kinks almost stays the same. Meanwhile, the kinks of the isolated wave stretch together in the clockwise and counterclockwise rotation, which makes the kinks of the isolated wave smoothen.

\section{Conclusion}

The exact traveling wave solutions obtained by the function expansion method are usually not the general traveling wave solutions of fractional-order nonlinear partial differential equations. In this paper, the fractional-order STO equation is transformed into an integer-order STO equation through the complex fractional-order transformation, and then, the general traveling wave solution of the fractional-order STO equation is obtained through the transformation of a specific function, which will make our understanding of the traveling wave solution of the fractional-order STO equation more comprehensive. By setting parameters, a kinked solitary wave solution is extracted from the general traveling wave solution, 
and the influence of the fractional derivative on the kinked solitary wave is analyzed in detail. It is found that the kinked solitary wave becomes more smooth when the fractionalorder parameters are synchronized, but the position of the kinked solitary wave is basically unchanged. The position of the kinked solitary wave is basically unchanged because we have two fractional-order parameters, one of which becomes smaller so that the kinked waveform rotates clockwise, and the other fractional-order parameter becomes smaller so that the kinked waveform rotates counterclockwise. Such clockwise rotation and counterclockwise rotation achieve a balance. The kink solitary wave becomes smoother because of the tugging phenomenon that accompanies the process of reaching equilibrium.

\section{Data Availability}

No data were used to support this study.

\section{Conflicts of Interest}

The authors declare that they have no conflicts of interest.

\section{Authors' Contributions}

All authors read and approved the final manuscript.

\section{Acknowledgments}

This research was supported by the Shanxi Provincial Key Research and Development Project (CN) (20181101008, 20181102015).

\section{References}

[1] H. Ç. Yaslan and A. Girgin, "Exp-function method for the conformable space-time fractional STO, ZKBBM and coupled Boussinesq equations," Arab Journal of Basic and Applied Sciences, vol. 26, no. 1, pp. 163-170, 2019.

[2] S. Bibi, S. T. Mohyud-Din, R. Ullah, N. Ahmed, and U. Khan, "Exact solutions for STO and $(3+1)$-dimensional KdV-ZK equations using G'G2-expansion method," Results in Physics, vol. 7, pp. 4434-4439, 2017.

[3] S. Sirisubtawee, S. Koonprasert, and S. Sungnul, "New exact solutions of the conformable space-time Sharma-Tasso-Olver equation using two reliable methods," Symmetry, vol. 12, no. 4 , p. 644,2020

[4] Y. W. Zhang, "Solving STO And KD equations with modified Riemann-Liouville derivative using improved (G'/G)-expansion function method," IAENG International Journal of Applied Mathematics, vol. 45, no. 1, pp. 16-22, 2015.

[5] A. Cesar and S. Gómez, "A nonlinear fractional SharmaTasso-Olver equation: new exact solutions," Applied Mathematics and Computation, vol. 266, pp. 385-389, 2015.

[6] H. Rezazadeh and F. S. Khodadad, "New structure for exact solutions of nonlinear time fractional Sharma-Tasso-Olver equation via conformable fractional derivative," Applications and Applied Mathematics: An International Journal, vol. 12, no. 1, pp. 405-414, 2017.

[7] R. Roy, M. A. Akbar, and A. M. Wazwaz, "Exact wave solutions for the nonlinear time fractional Sharma-Tasso-Olver equation and the fractional Klein-Gordon equation in mathematical physics," Optical and Quantum Electronics, vol. 50, no. 1, pp. 1-19, 2018.

[8] R. Khalil, M. al Horani, A. Yousef, and M. Sababheh, "A new definition of fractional derivative," Journal of Computational and Applied Mathematics, vol. 264, pp. 65-70, 2014.

[9] A. Atangana, D. Baleanu, and A. Alsaedi, "New properties of conformable derivative," Open Mathematics, vol. 13, no. 1, pp. 889-898, 2015.

[10] W. Chen, "Time-space fabric underlying anomalous diffusion," Chaos, Solitons and Fractals, vol. 28, no. 4, pp. 923929, 2006.

[11] W. Cai, W. Chen, and W. Xu, "Characterizing the creep of viscoelastic materials by fractal derivative models," International Journal of Non-Linear Mechanics, vol. 87, pp. 5863, 2016.

[12] D. Z. Zhao and M. Luo, "General conformable fractional derivative and its physical interpretation," Calcolo, vol. 54, no. 3, pp. 903-917, 2017.

[13] T. Abdeljawad, "On conformable fractional calulus," Journal of Computational and Applied Mathematics, vol. 264, pp. 65-70, 2015.

[14] M. N. Alam and M. S. Osman, "New structures for closedform wave solutions for the dynamical equations model related to the ion sound and Langmuir waves," Communications in Theoretical Physics, vol. 73, no. 3, article 035001, 2021.

[15] M. N. Alam, E. Bonyah, M. Fayz-al-Asad, M. S. Osman, and K. M. Abualnaja, "Stable and functional solutions of the Klein-Fock-Gordon equation with nonlinear physical phenomena," Physica Scripta, vol. 96, no. 5, article 055207, 2021.

[16] F. S. Bayones, K. S. Nisar, K. A. Khan et al., "Magneto-hydrodynamics (MHD) flow analysis with mixed convection moves through a stretching surface," AIP Advances, vol. 11, no. 4, article 45001, 2021.

[17] M. Yang, M. S. Osman, and J. G. Liu, “Abundant lumptype solutions for the extended (3+1)-dimensional JimboMiwa equation," Results in Physics, vol. 23, article 104009, 2021.

[18] S. Kumar, B. Kour, S. W. Yao, M. Inc, and M. S. Osman, "Invariance analysis, exact solution and conservation laws of $(2+1)$ dim fractional Kadomtsev-Petviashvili (KP) system," Symmetry, vol. 13, no. 3, p. 477, 2021.

[19] M. Akher Chowdhury, M. Mamun Miah, H. M. Shahadat Ali, Y. M. Chu, and M. S. Osman, "An investigation to the nonlinear $(2+1)$-dimensional soliton equation for discovering explicit and periodic wave solutions," Results in Physics, vol. 23, p. 104013, 2021.

[20] S. Javeed, S. Saif, A. Waheed, and D. Baleanu, "Exact solutions of fractional mBBM equation and coupled system of fractional Boussinesq-Burgers," Results in Physics, vol. 9, pp. 1275-1281, 2018.

[21] N. A. Kudryashov, "Seven common errors in finding exact solutions of nonlinear differential equations," Communications in Nonlinear Science and Numerical Simulation, vol. 14, no. 9-10, pp. 3507-3529, 2009.

[22] N. A. Kudryashov, "On "new travelling wave solutions" of the $\mathrm{KdV}$ and the KdV-Burgers equations," Communications in Nonlinear Science and Numerical Simulation, vol. 14, no. 5, pp. 1891-1900, 2009. 
[23] M. N. Ali, S. M. Husnine, A. Saha, S. K. Bhowmik, S. Dhawan, and T. Ak, "Exact solutions, conservation laws, bifurcation of nonlinear and supernonlinear traveling waves for SharmaTasso-Olver equation," Nonlinear Dynamics, vol. 94, no. 3, pp. 1791-1801, 2018.

[24] K. Fan and C. Zhou, "Mechanical solving a few fractional partial differential equations and discussing the effects of the fractional order," Advances in Mathematical Physics, vol. 2020, Article ID 3758353, 17 pages, 2020. 